\title{
Study Of Quality And Reliability Of Welding Process Reflow Component Technology BGA
}

\author{
Robson Marques Costa ${ }^{1 *}$, Jandecy Cabral Leite ${ }^{2 *}$, Antonio da Silva Vieira ${ }^{1}$, Raimundo Valdan Pereira Lopes ${ }^{1}$, Roberto \\ Tetsuo Fujiyama ${ }^{1}$ \\ ${ }^{1}$ Programa de Pós Graduação em Engenharia Industrial-PPGEI/Universidade Federal do Pará-UFPA. Rua Augusto Correa 01 \\ Guamá. Belém - Pará - Brasil. CEP: 66075-110. Fone: +55 (92) 33215643 ou +55 (92) 91292218. \\ ${ }^{2}$ Instituto de Tecnologia e Educação Galileo da Amazônia - ITEGAM Praça Francisco Pereira da Silva, 149, Bola da Suframa - \\ Distrito Industrial. Manaus - Amazonas - Brasil. CEP: 69073-270. Fone: +55 (92) 3639-8952 ou +55 (92) $3584-6145$. \\ *robson_m_costa@hotmail.com,*jandecy.cabral@itegam.org.br
}

\begin{abstract}
In recent years many efforts have been expended by companies in the search for technological improvement of its products by adding features such as light weight, reduced size and high levels of performance at the lowest cost, to meet a worldwide demand in this regard. This article aimed to conduct an analysis of the quality and reliability of the Reflow soldering process of component of BGA technology. The methods and techniques used were the qualitative-quantitative approach, conducted by the case study technic in the welding process of the BGA component by collecting (cross section and X-ray) and data analysis (alignment, cracks and voids) within the process. The achieved results showed that the Reflow welding process of BGA component meets the criteria for acceptance of international standards IPC -A - 610E and IPC 7095B. This fact leads to the inference that the quality of the process in question may reflect in better conditions and cost competitiveness for the investigated organization.
\end{abstract}

Keywords: Quality in the process, Reflow Soldering, BGA component, Cross section, Cracks and Voids.

\section{Study Of Quality And Reliability Of Welding Process Reflow Component Technology BGA}

\begin{abstract}
RESUMO
Atualmente muitos esforços vêm sendo despendido por empresas na busca por aperfeiçoamento tecnológico dos produtos agregando aspectos como leveza, redução de dimensão e altos níveis de desempenho ao menor custo, visando atender uma demanda mundial. Este artigo objetivou realizar um estudo de qualidade e confiabilidade do processo Reflow de soldagem de componente de tecnologia BGA. Os métodos e técnicas utilizadas foram a abordagem qualitativo-quantitativo, orientado mediante técnica de estudo de caso no processo de soldagem de componente BGA mediante coleta (cross section) e análise de dados (alinhamento, cracks). Os resultados obtidos demonstraram que o processo Reflow de soldagem do componente BGA atende a critérios de aceitabilidade de normas internacionais IPC-A 610E e IPC-7095B. Tal fato leva a inferir que a qualidade do processo em questão pode refletir em melhores condições de custo e de competitividade para a organização investigada.
\end{abstract}

Palavras Chaves: Qualidade no processo, Soldagem Reflow; Componente BGA, Cross section, Cracks.

\section{INTRODUÇÃO}

A revolução recorrente nos dispositivos eletroeletrônicos nos últimos anos é motivada pela agregação de inteligência as funcionalidades destes dispositivos. A evolução da tecnologia nos componentes eletroeletrônicos destes produtos acarreta por efeito uma miniaturização dos componentes e aumento no desempenho de funcionamento, obedecendo a tendência em dispositivos eletrônicos em direção a dispositivos menores e mais leves, com aumento de funcionalidades [1], contudo, a miniaturização torna a montagem e soldagem destes componentes mais complexa, exigindo um nível de controle 
e confiabilidade do processo cada vez maior.A empresa pesquisada apresenta em seu processo Produtivo de Placas de Circuito Impresso Mother Board (PCI MB) de Laptops uma etapa em que é realizada a soldagem Reflow de componentes de tecnologia BGA, e pode-se dizer que é difícil verificar e alertar as falhas anteriormente, porque as falhas são causadas por vários fatores, tais como termo-mecânicos e estresses de choque [2], entretando, a empresa desconhece o nível de qualidade e confiabilidade do processo de soldagem Reflow realizado neste processo, limitando-se somente a verificar presença de Voids nos BGA, e tal conhecimento do processo é importante, pois quanto mais uma organização investe em medidas preventivas mais eles são capazes de reduzir os custos de falhas e a consequente otimização de custos da qualidade gera oportunidade de maior lucro mantendo o mesmo preço para o produto [3].

Logo, o objetivo deste artigo foi realizar uma pesquisa que diagnosticasse a qualidade e confiabilidade do processo de soldagem Reflow de componente de tecnologia BGA após a realização da soldagem Reflow do componente em PCI's MB de Laptop em uma empresa do Polo Industrial de Manaus (PIM). A empresa em estudo ao longo do ano de 2013 passou por um processo de migração de Tecnologia de Solda Tin-Lead para Lead-Free, atendendo uma demanda internacional, no entanto, esta transição agrava os problemas de processo, pois as ligas lead-free são geralmente mais duras e mais quebradiço do que soldas de chumbo-estanho convencionais [4]. Uma das consequencias não intencional desta migração é que a indústria teve que recorrer a novos métodos para a soldagem de componentes nas placas, isto vem em um momento em que a indústria de semicondutores está desenvolvendo componentes continuamente menores e acomodando maiores funcionalidades [5].

A pesquisa em questão justifica-se pela necessidade de um diagnóstico do processo de soldagem em fase de implantação, pois o uso de materiais e processos isentos de chumbo, também leva a novas preocupações com a confiabilidade [6], tornando-se este tipo de pesquisa imprescindível para quaquer organização que prentenda oferecer a seus clientes um produto de qualidade, evitando assim que a empresa assuma riscos de confiabilidade de seus produtos ao dispo-los no mercado, e podendo tornar-se tal pesquisa referencia para trabalhos futuros dentro da organização.

\section{REVISÃO BIBLIOGRÁFICA 2.1 Ball Grid Array (BGA)}

Componentes do tipo Ball Grid Array (BGA) são comumente usado em indústria de microeletrônica que trabalham com alta densidade pino (terminais), boa condução de calor e baixo custo [7]. A Tecnologia BGA está entre os mais atraentes e amplamente utilizados opções de encapsulamento por causa de muitos benefícios, como a redução de problemas de coplanaridade, redução do tamanho, melhor performances elétrica e térmica etc [8].

O BGA é utilizado em vários tipos de componentes, entre eles chipsets e chips de memória. É um tipo de componente onde os terminais de contato do tipo esfera. A Figura 1 mostra um componente tipo BGA e seus Balls. a)

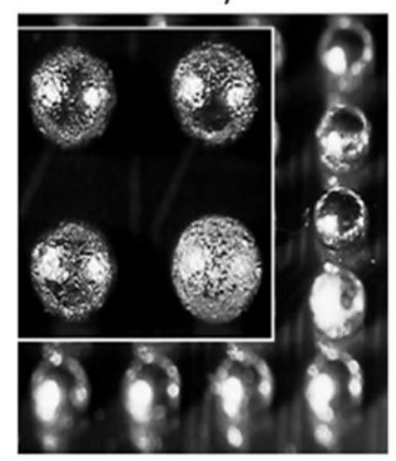

b)

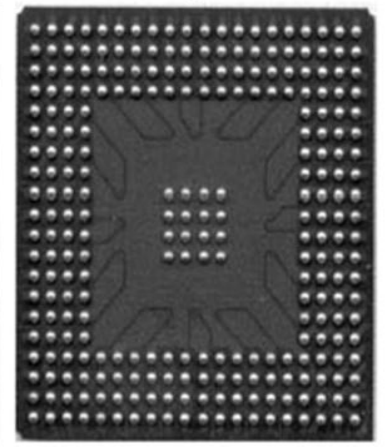

Figura 1: a) Esferas do componente (Ball) Liga $\mathrm{SnAgCu}$; b) Componente BGA.

A placa $\mathrm{MB}$ que recebe este componente passa pelo processo SMT típico: Impressão de Pasta de Solda, procedimento de aplicação de pasta de solda montagem do componente (Place Component) e soldagem no forno de refusão. Após a soldagem do BGA na PCI as esferas (Balls) não ficam visíveis. A Figura 2 mostra o fluxo do processo SMT para o componente BGA [9], neste processo (1) mostra a PCB, (2) a printagem da solda em pasta de solda sobre a PCB, (3) a montagem do componente BGA e (4) a soldagem do componente.

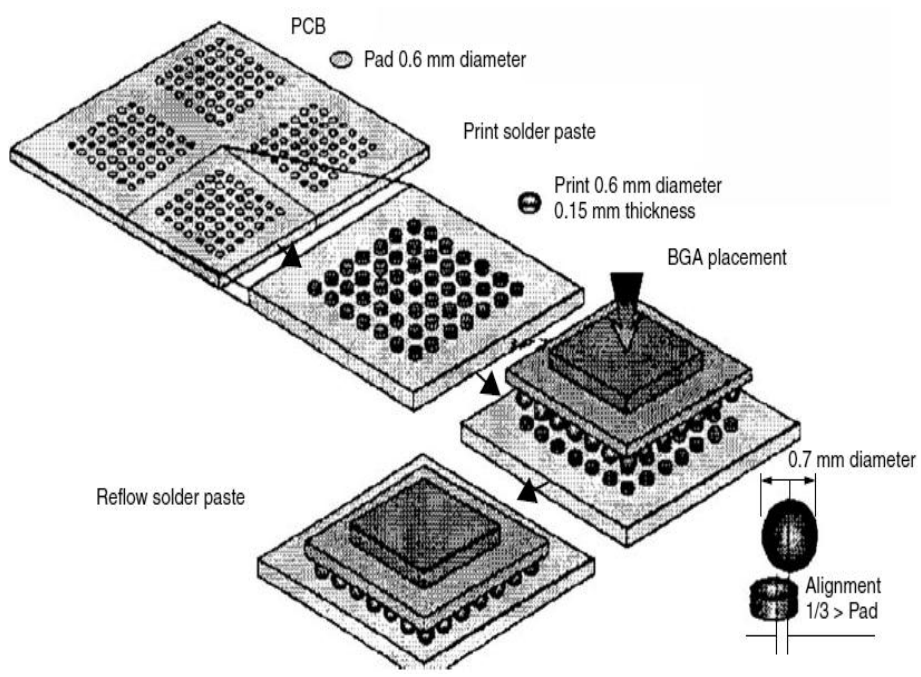

Figura 2: Fluxo do processo de montagem de um BGA. 


\subsection{Processo Reflow}

O perfil de tempo- temperatura de Reflow é de grande importância no processo de soldagem de forno de refusão. O perfil é a relação de temperatura com respeito ao tempo necessário para levar a solda da montagem de PCI do estado líquido e de volta ao estado sólido antes de sair do forno [10]. O Processo de Reflow é uma parte do processo de Tecnologia de Montagem sobre Superficie (SMT), tecnologia onde os componentes são montados diretamente sobre a superfície da placa de circuito impresso [11].

Processos de Linhas de montagem SMT geralmente envolvem pasta de solda, e colocação dos componentes. O processo Reflow de soldagem consiste em aquecer toda a placa montada para liquefazer a pasta de solda para formar juntas de solda entre os SMDs e o PCB sem alterar as características originais dos componentes elétricos, sendo um forno de refusão utilizado para aquecer as placas montadas a uma temperatura apropriada em um especificado período de tempo a uma taxa de aquecimento adequado [13]. Um modelo característico de perfil recomendado é descrito na Figura 3 [13]:

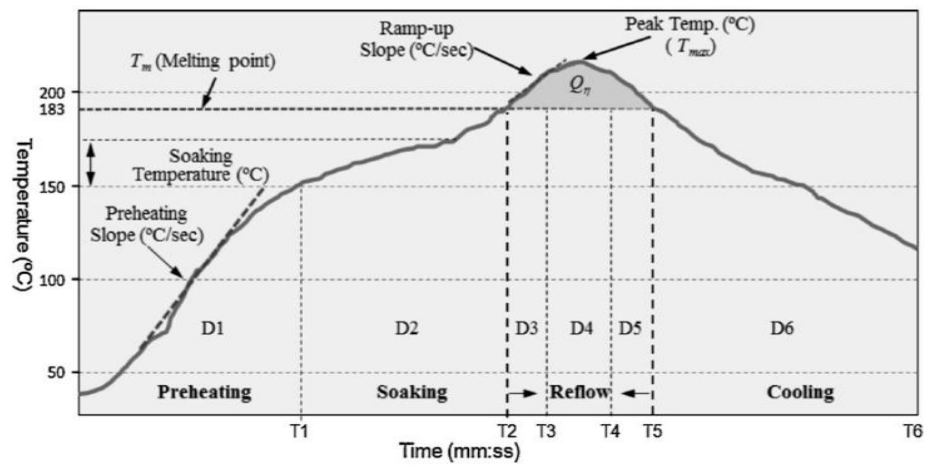

Figura 3: a) Perfil de temperatura típico de Reflow

\subsection{Norma IPC}

A IPC - Association Connecting Electronics Industries é uma associação comercial global dedicada a excelência competitiva de suas empresas filiadas ao redor do mundo. A IPC serve a indústria de eletrônicos, voltada especificamente para os fabricantes relacionados de alguma forma ao processo de Placas Impressas com padrões de aceitabilidade internacionalmente aceitos [14]. IPC Normas e Publicações são projetados para servir o interesse público através da eliminação de malentendidos entre fabricantes e compradores, facilitando a intercambialidade e melhoria de produtos, e auxiliando o comprador na seleção e obtenção com atraso mínimo do produto adequado para sua necessidade particular [15]. Dentre as Certificações existentes na IPC, este artigo utilizou a IPC-7095B, cujo foco sobre as informações estão em inspeção crítica, reparo e problemas de confiabilidade associados BGAs [15].

\subsection{Implementação de Solda Lead-free}

A União Europeia aprovou uma legislação, a Diretiva RoHS (Restriction of Hazardous Substances), que proíbe o uso de chumbo $(\mathrm{Pb})$ e várias outras substâncias em produtos eletrônicos com início de julho de 2006 [16]. Deste ano em diante o uso de um dos componentes mais utilizados na solda, o chumbo, não seria mais são usados em solda eletronica devido à legislação, entre alternativas sem chumbo, a solda lead free $(\mathrm{SnAgCu})$ é um substituto amplamente aceito, devido à sua melhor fluência fadiga resistência e a estabilidade microestrutural [17].

$\mathrm{Um}$ dos principais diferenças entre $\mathrm{SnPb}$ e $\mathrm{SnAgCu}$ soldas sem chumbo é que soldas $\mathrm{SnAgCu}$ exigem maior temperatura de Reflow que soldas $\mathrm{SnPb}$, o ponto de fusão é 217 ${ }^{\circ} \mathrm{C}-219{ }^{\circ} \mathrm{C}$, superior ao da solda $\mathrm{SnPb}$, que tem um ponto de fusão de $183{ }^{\circ} \mathrm{C}$ [18].

Também é necessário verificar a adequação dos componentes de montagem, devem ser tomadas medidas para proteger os componentes contra dano potencial provocados pelas temperaturas de Reflow superior e a qualidade e confiabilidade de juntas e materiais de solda e sua adequação para uma determinada aplicação deve ser verificada [19]. O componente BGA é um exemplo amplamente utilizado em placa de circuito impresso por processo de soldagem reflow, o qual é um dos itens crítico em tecnologia de montagem em superfície. Junta de solda quebra é um dos principais modos de falha devido ao processo lead free de solda reflow inadequado [20]. Atualmente empresas brasileiras que exportam para a União Europeia, países que adotaram a Rohs e que participam de programas de licitação nacional já migraram para uma tecnologia de solda sem chumbo.

\section{MATERIAIS E MÉTODOS}

A seguir descrevem-se os principais aspectos metodológicos empregados na elaboração deste estudo de caso, que teve como foco central o diagnóstico estudo de nível de qualidade e confiabilidade do processo de soldagem realizada na troca de componentes de tecnologia BGA.

\subsection{Natureza da Pesquisa}

Este trabalho pode ser classificado como uma pesquisa descritiva, que utiliza a técnica do estudo de caso como ferramenta de coleta e análise de dados, com uma abordagem tanto qualitativa quanto quantitativa, o método do estudo de caso 
Robson Marques Costa, et al / ITEGAM-JETIA Vol.01, N ${ }^{\mathrm{o}}$ 01, pp.35-40. Março, 2015.

permite que os investigadores retenham as características holísticas e significativas dos eventos da vida real [21].

\subsection{Definições da oportunidade da Pesquisa}

O uso de componentes de tecnologia BGA necessita por parte das empresas de um complexo monitoramento do processo para assegurar a confiabilidade de soldagem. Esta pesquisa foi desenvolvida com base no processo de soldagem Reflow de componentes de tecnologia BGA, teve como objetivo realizar um estudo para diagnosticar a qualidade e confiabilidade da soldagem neste processo. Optou-se por realizar um estudo deste processo de soldagem de BGA, pois esta fase caracteriza-se como uma etapa complexado processo dada a miniaturização dos componentes. Os BGAs evoluíram como uma solução viável para a exigência da indústria e com este tipo de componente, a confiabilidade da junta de solda se tornou um dos fatores mais críticos do processo [22].

\subsection{Das Etapas}

Para o estudo de caso, utilizou-se de revisão de literatura sobre os conceitos fundamentais relacionados ao tema, busca de artigos recentes sobre aplicação similares e Normas técnicas Internacionais IPC-A-610E e a IPC-7095B para diagnosticar a aceitação da soldagem do tipo de componente estudado. Inicialmente, buscou-se uma fundamentação teórica por meio de artigos científicos onde se verificou que o ensaio cross section é largamente utilizado para este tipo de estudo. O ensaio de cross section foi realizado em um laboratório de reconhecimento nacional e os dados do ensaio foram tabulados assim como as imagens fotograficas do componente obtidas por meio de microscópio eletronico.

\subsection{Delimitação da pesquisa}

Estudos de confiabilidade de soldagem têm vasta aplicação em diversos componentes no são mais comumente utilizados para avaliação da soldagem: O Cross-section e o Dye \& Pry. Este artigo está delimitado ao estudo de um componente de tecnologia BGA de posição mecânica U22 da PCI MB de um Notebook e o tipo de no segmento eletroeletrônico e dois tipos ensaios são mais comumente utilizados para avaliação da soldagem: ensaio mecânico que será realizado é o Cross-section que mostrará características dos BGA's situados no canto direito, no canto esquerdo e no centro do componente. Como delimitação temporal, as amostras de PCI MB foram coletadas entre Abril e Maio 2013, de tal forma que possibilitou um acompanhamento do desempenho do processo em períodos sequências.

\section{RESULTADOS E DISCUSSÕES}

Esta pesquisa foi desenvolvida com base no estudo do processo de soldagem Reflow de componentes de tecnologia BGA avaliando a qualidade e confiabilidade da soldagem. Esta atividade esta inserida no processo de fabricação de Notebooks e a pesquisa foi realizada no período de 01 de Abril a 31 de Maio de 2013. Conforme descrito na metodologia, foi delimitado nesta a)

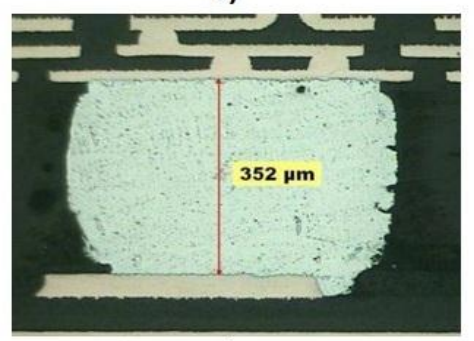

c)

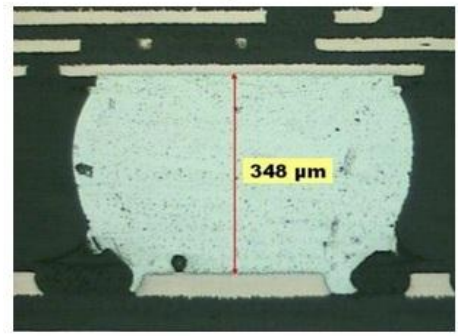

b)

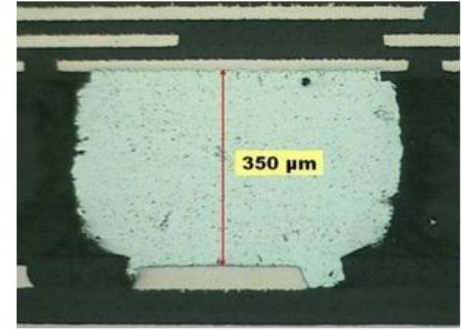

d)

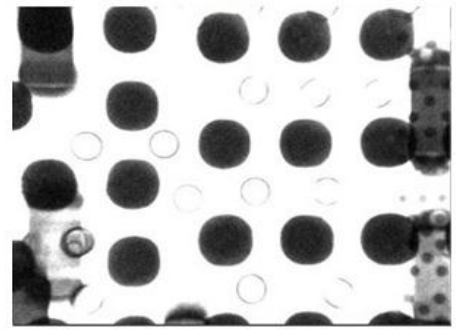

pesquisa como ensaio de avaliação o método Cross-section do BGA. O ensaio cross section foi realizado externamente em um laboratório de análises credenciado e os dados obtidos no componente BGA a partir deste ensaio são mostrados na Figura 5 .

Figura 5: Cross Section e Raio X: a) Ball do canto Esquerdo U22; b) Ball do canto Direito U22; c) Ball do centro U22 e d) Raio X do BGA.

A Figura 5 mostra respectivamente as imagens obtidas com a ampliação $100 \mathrm{X}$ por microscópio óptico do corte realizado no Ball do BGA no canto esquerdo (a), canto direito (b), Ball do centro (c) e (d) componente BGA em estudo.

\subsection{Análise dos Resultados}

Analisando os resultados da Figura 5 com base nos requisitos da IPC A-610E e IPC-7095B é possível constatar que:

i) O alinhamento do componente é considerado aceitável.

O posicionamento do solder ball do BGA é centrado e não mostra nenhum deslocamento do Ball para os centros de Pad [14]. A Figura 6 mostra imagem de alinhamento do Ball segundo a IPC A-610E. 
ii) Não foram encontrados rachaduras (cracks) nos Balls.

A Figura 6 mostra o crack de um ball especificado pela IPC A-610E, um crack de um ball e um ball sem crack segundo a IPC-7095B.

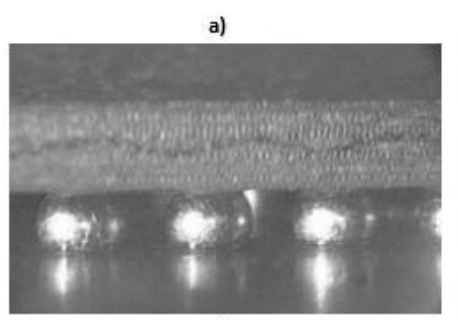

c)

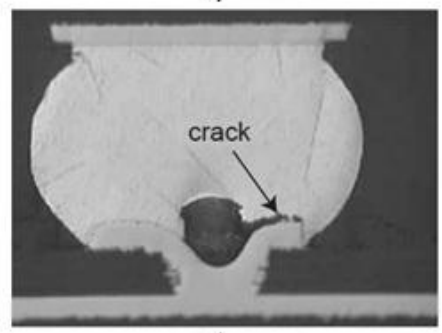

Figura 6: Critérios de aceitabilidade IPC: a) Alinhamento; b) Crack IPC A-610E; c) Crack IPC-7095B; d) No Cracks IPC-7095B;

Como base nos dados (i-ii) verificados na análise crossection do componente BGA segundo critérios de aceitabilidade mostrados na Figura 6 é possível afirmar que a junta de solda analisada foi encontrada em conformidade com a Norma IPC-A610 e IPC-7095B, portanto, pode ser dito que possui confiabilidade de solda em suas juntas, logo o processo de soldagem Reflow de componentes BGA atende conceitos internacionais de confiabilidade.

\section{CONCLUSÃO}

Com base na análise dos resultados mostrados na Figura 5, é possível afirmar que a empresa pode realizar o processo de soldagem Reflow de componentes BGA, inserido no processo de fabricação de notebooks, sem por em risco a qualidade final do produto, pois o estudo de crosss-section realizado no BGA demonstrou que o processo de soldagem realizado atende os critérios de aceitabilidades das Normas Internacionais IPC-A610E e IPC-7095B, portando, pode-se concluir que há qualidade e confiabilidade no processo de soldagem Reflow de componente de tecnologia BGA.

Ter conhecimento da qualidade e confiabilidade de seus processos é essencial para que a empresa possa trabalhar para obter menores custos e melhores condições de competitividade, logo, este artigo pôde demonstrar a importância da confiabilidade dos processos de soldagem Reflow de componentes BGA tornando-se referência para trabalhos futuros para organizações do segmento eletroeletrônico que utilizam componentes BGA em seu processo de fabricação.

\section{AGRADECIMENTOS}

A Universidade Federal do Pará (UFPA), ao Instituto de Tecnologia e Educação Galileo da Amazônia (ITEGAM) e o Instituto Tecnologia José Rocha Sergio Cardoso (ITJRSC)

\section{REFERÊNCIAS BIBLIOGRÁFICAS}

[1] Lee, Yong-Won. et al. The Effect of Microvia-In-Pads Design on SMT Defects in Ultra-Small Component Assembly. International Conference on Electronic Packaging Technology \& High Density Packaging. IEEE. 2010.

[2] Liu, Cang. et al. Research on the fault diagnosis technology of intermittent connectionfailure belonging to FPGA solderjoints in BGA package. Optik optics, n.125, p. 737- 740. 2014.

[3] Priede, Janis. Implementation of Quality Management System ISO 9001 in the World and its Strategic Necessity. Procedia. Volume 58, p. 1466-1475. 2012.

[4] Yang, Chaoran. et al. Comparative Study of PWB Pad Cratering Subject to Reflow Soldering and Thermal Impact. Electronic Components and Technology Conference, p. 464-470. IEEE. 2010.

[5] Childs, Joe. How Lead-Free Changes Can Impact Reliability. Annual Technology Report. IEEE, 2008.

[6] Ganesan, S \& Pecht, M. Lead-Free Electronics. College Park, MD: CALCE EPSC Press, 2004.

[7] Wang, Fuliang. et al. Height Measurement of Micro-Solder Balls on Metal Pad by White Light Projection. IEEE Transactions on Components, Packaging and Manufaturing Technology. Vol.2. n 9. 2012.

[8] Stoyanov, Stoyan. et al. Thermo-Mechanical SubModelling of BGA Components in PCB Reflow. IEEE. 36th Int. Spring Seminar on Electronics Technol, p. 253- 258. 2013.

[9] Lee, Ning-Cheng. Reflow Soldering Processes and Troubleshooting: SMT, BGA, CSP and Flip Chip Technologies. Newnes. 2002. 
[10] Freedman, Gary M. Chapter 44: Soldering Fundamentals, in Coombs, Clyde F. Jr: Coomb's Printed Circuits Handbook. Sixth Edition, McGraw-Hill. 2008.

[11] Jirsa, Jan. et al. Risk Analysis of Reflow Technologies in Electronics Assembly. IEEE. 35th Int. Spring Seminar on Electronics Technology, p. 178-182. 2012.

[12] Ayob, Masri \& Kendall, Graham. A survey of surface mount device placement machine optimisation: Machine classification. European Journal of Operational Research, n.186, p. 893-914. 2008.

[13] Tsai, Tsung-Nan. Thermal parameters optimization of a reflow soldering profile in printed circuit board assembly: A comparative study. Applied Soft Computing, n.12, p. 2601-2613. 2012.

[14] IPC-A-610E. Acceptability of Electronic Assemblies. 2010.

[15] IPC-7095B. Design and Assembly Process Implementation for BGAs. 2008.

[16] Puttlitz, Karl J. \& Galyon, George T. Impact of the ROHS directive on high-performance electronic systems. J Mater Sci: Mater Electron, n.18, p.331-346. 2006

[17] Schreurs, P.J.G. et al. Integrated numerical-experimental analysis of interfacial fatigue fracture in $\mathrm{SnAgCu}$ solder joints. International Journal of Solids and Structures, n.44, p. 5680-5694. 2007

[18] Pan, Jianbiao. et al. Effect of Reflow Profile on SnPb and SnAgCu Solder Joint Shear Force. APEX. 2006.

[19] Yamamoto, Tsuyoshi \& Tsubone, Ken-ichlro. Assembly Technology Using Lead-free Solder. FUJITSU Sei. Tech, n. 43, p.50-58. 2007

[20] Lau, Chun-Sean. et al. Effect of Solder Joint Arrangements on BGA Lead-Free Reliability During Cooling Stage of Reflow Soldering Process. IEEE Transactions on Components, Packaging and Manufacturing Technology, Vol. 2, N.12, p. 2098-2107. 2012

[21] Yin, Robert K. Case Study Research: Design and Methods. SAGE. Fourth Edition. 2009

[22] Lin, Weicheng. The Void-free Reflow Soldering of BGA with Vacuum. IEEE. 2007. 